\title{
Phosphorylation of AMMECR1 at Serine16 Is Not Essential for Its Nuclear Localization
}

\author{
Chengfeng Cai ${ }^{1,2 *}$, Meng $\mathrm{Xu}^{2 *}$, Yongquan Su${ }^{1,3}$, Huamin Zhou ${ }^{2 \#}$ \\ ${ }^{1}$ State Key Laboratory of Large Yellow Croaker Breeding, Fujian Fuding Seagull Fishing Food Co., Ltd., Fuding, China \\ ${ }^{2}$ State Key Laboratory of Cellular Stress Biology, School of Life Sciences, Xiamen University, Xiamen, China \\ ${ }^{3}$ College of Ocean and Earth Sciences, Xiamen University, Xiamen, China \\ Email: "huaminzhou@xmu.edu.cn
}

How to cite this paper: Cai, C.F., Xu, M., Su, Y.Q. and Zhou, H.M. (2019) Phosphorylation of AMMECR1 at Serine16 Is Not Essential for Its Nuclear Localization. Journal of Biosciences and Medicines, 7, 146-153.

https://doi.org/10.4236/jbm.2019.711013

Received: October 27, 2019

Accepted: November 24, 2019

Published: November 27, 2019

\begin{abstract}
$A M M E C R 1$ is a critical gene in the pathogenesis of AMME syndrome. But, little is known about how AMMECR1 is regulated. Here, we showed that many human cell lines expressed AMMECR1. With Phos-tag SDS PAGE analysis, we demonstrated that AMMECR1 was constitutively phosphorylated at Ser16. AMMECR1 was localized in the nucleus. Mutation of Ser16 to alanine did not affect its nuclear localization. The homologs of AMMECR1, PAC688.03c and MTH857, were also nuclear proteins, but they were not phosphorylated when tested in HeLa cells. Therefore, AMMECR1 and its homologs might have atypical nuclear localization sequences.
\end{abstract}

\section{Keywords}

AMMECR1, Transcription, Phosphorylation, Nuclear Localization

\section{Introduction}

AMME (Alport syndrome, Mental retardation, Midface hypoplasia, and Elliptosis) syndrome is a pathogenic condition that is caused by a deletion of a fragment of $\mathrm{X}$ chromosome [1] [2]. AMMECR1 (AMME chromosomal region gene 1) is one of the genes in this region. Andreoletti et al. (2017) reported that a single missense mutation in $A M M E C R 1$ (c.G530A; p.G177D) alone causes a phenotype of midface hypoplasia, speech and language delay and elliptocytosis [3]. A loss-of-function of the $\mathrm{X}$-linked $A M M E C R 1$ in five individuals is associated with growth, bone, and heart alterations [4]. The mutation (C502T) in the proband results in a substitution of an Arginine to a termination codon, which causes alternative splicing of AMMECR1 mRNA that "skips" exon 2 to lead to ${ }^{\star}$ Both authors contributed equally to this work. 
an in-frame deletion responsible for the elliptocytosis with or without anemia, midface hypoplasia, proportionate short stature and hearing loss [5]. Thus, $A M M E C R 1$ is an important gene in the pathogenesis of AMME.

However, the biological function of AMMECR1 is unknown [6]. AMMECR1 has not been studied in cultured cell yet. Here, we found that $A M M E C R 1$ is ubiquitously expressed in many human cell lines. AMMERC1 is a nuclear protein and constitutively phosphorylated at Ser16, but phosphorylation of Ser16 is not required for its nuclear localization.

\section{Materials and Methods}

\subsection{Reagents}

$\lambda$-Phosphatase ( $\lambda$ PPase) was purchased from New England Biolabs. Phos-tag ${ }^{\text {TM }}$ AAL-107 was from Wako Pure Chemical Industries, Ltd. The following antibodies were purchased: anti-FLAG monoclonal antibody affinity gel M2 from Sigma; anti-flag monoclonal antibodies from ABclonal Biotechnology, horseradish peroxidase-labeled goat anti-mouse or anti-rabbit IgG secondary $A b$ from Pierce.

\subsection{DNA Constructs}

$A M M E C R 1$ ( $S$ ) cDNA was from Han Lab cDNA library. SPAC688.03c gene was cloned from $S$. pombe. MTH857 gene was synthesized. AMMECR1 mutations were introduced by site-directed mutagenesis using the QuickChange Kit from Stratagene (La Jolla, CA). All plasmids were verified by automated sequence analysis.

\subsection{Cell Culture and Transfection}

All cells were cultured in Dulbecco's modified Eagle's medium supplemented with $10 \%$ fetal calf serum (Hyclone) and $100 \mu \mathrm{g} / \mathrm{ml}$ penicillin and streptomycin at $37^{\circ} \mathrm{C}$ in a humidified incubator with $5 \% \mathrm{CO}_{2}$. Cells were transiently transfected with $2 \mu \mathrm{g}$ (total) of plasmids using Lipofectamine 2000 transfection reagent (Invitrogen) and PEI (Invitrogen) for HEK293T and HeLa, respectively.

\subsection{Measurement of mRNA Expression}

For 3' Race, total RNA was isolated using Trizol reagent (Invitrogen) and converted into $\mathrm{CDNA}$ with $\mathrm{RT}$ primer

(5'-ccagtgagcagagtgacgaggactcgagctcaagctcaggaataatggt tgtatg-3'). A nested PCR was performed with the resultant cDNA by using L2/Q1 as first round primer pair (L2:5'-tcttcctcaccctcctcatc-3'/Q1:5'-ccagtg agcagagtgacg-3'), L3/Q2

(L3:5-gattcaccaacgagcctat-3'/Q2:5'-cgaggactcgagctcaag ct-3') and L4/Q2 (L4:

5'-gcaccgccacctacctac-3') as second round primer pairs, respectively. L2 - L4 are located on the AMMECR1 gene, whereas $\mathrm{Q} 1$ and $\mathrm{Q} 2$ are on the anchor sequence of RT primer. The PCR fragments were cloned into the pBKS (+) vector. Their sequences were verified by automated sequencing analysis. 


\subsection{Immunoprecipitation and Western Blotting}

Cells were lysed in lysis buffer with $1 \%$ Triton X-100, protease inhibitors, and incubated with anti-flag M2 beads and gently shaken for $4 \mathrm{~h}$ at $4^{\circ} \mathrm{C}$. The beads were washed three times with the lysis buffer. The precipitates and total cell lysates were resolved on Phos-tag SDS PAGE or conventional SDS PAGE, transferred to PVDF membranes, and then immunoblotted with primary Abs anti-flag and the secondary $\mathrm{Ab}$ conjugated with horseradish peroxidase. The presence of a protein band was visualized using enhanced chemiluminescence (ECL) detection reagents. Phos-tag SDS-PAGE gel was prepared according to the manufacturer's protocol.

\section{6. $\lambda$ PPase Dephosphorylation}

The $50 \mu \mathrm{L}$ reaction mixture was supplemented with $200-400$ units of $\lambda$ PPase and the reaction was allowed to proceed for $30 \mathrm{~min}$ at $30^{\circ} \mathrm{C}$ according to manufacturer's instructions.

\section{Results and Discussion}

\subsection{Transcriptional Analysis of AMMECR1 in Cell Lines}

$A M M E C R 1$ is mainly transcribed in two isoforms, as illustrated in Figure 1(A) [2]. The long isoform is $1002 \mathrm{bp}$ long (called $A M M E C R 1$ ), whereas the shorter form is $891 \mathrm{bp}$ (not containing the conservative coding region for LRGCIG,

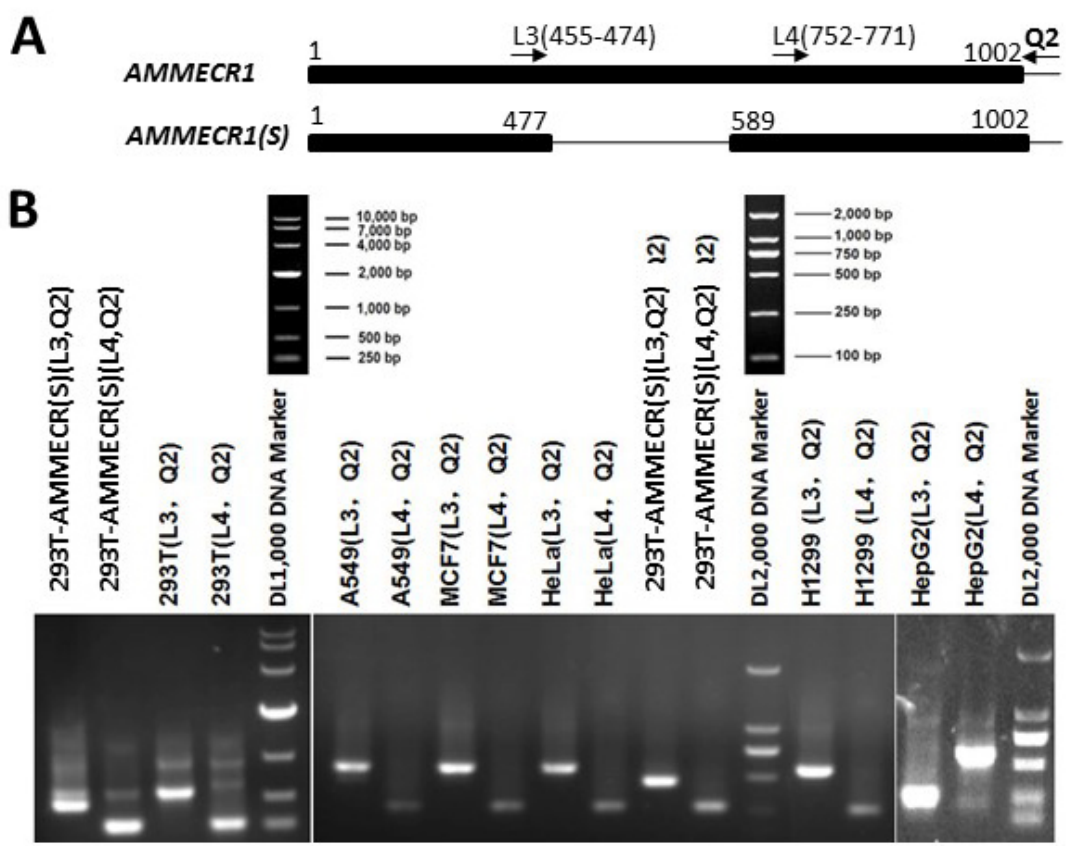

Figure 1. Expression of $A M M E C R 1$ gene in different cell lines. The presence of $A M M E C R 1$ transcripts in a variety of cell lines was ascertained by nested PCR. The fragment sizes with the primer pair L3/Q2 was 568 bp for AMMECR1, 457 bp for AMMECR1 $(S)$, whereas that with the primer pair L4/Q2 was $271 \mathrm{bp}$ for both. The positions of size markers were indicated. 
called $A M M E C R 1$ ( $S$ ) thereafter). To explore the expression pattern of these two isoforms in human cell lines including HEK293T, MCF7, A549, H1299, HeLa and HepG2, we designed a series of nested PCR forward primers from the AMMECR1 coding region. cDNAs were isolated from HEK293T cells which were transfected with pcDNA-AMMECR1 (S) and used as positive control. With second round nested PCR primer pairs L3/Q2 or L4/Q2 (Figure 1(A)), a clear single band was observed. When the primer pair L4/Q2 was used, all the cell lines showed the same size of amplification fragments as $A M M E C R 1(S)$; however, when L3/Q2 primer pair was used, all cells showed a longer fragment than $A M M E C R 1(S)$, equivalent to $A M M E C R 1$ (Figure 1(B)). These results indicate that all of these cells expressed the long isoform of $A M M E C R 1$. So we will select $A M M E C R 1$ isoform mainly for study in this paper.

Although $A M M E C R 1$ was expressed in many cell lines and generally widely distributed in tissue, but the mouse trophoblast stem cell K4GFP do not express $A M M E C R 1$ [7]. On the other hand, the high expression of $A M M E C R 1$ (S) will lead to the disease [5].

\subsection{AMMECR1 Is Nuclear Protein}

Protein nuclear localization is generally related to its biological function [8] [9]. Although there is no special verification signal sequence in AMMECR1, but it still is nucleoprotein [2] [3] [4]. Our result showed also that AMMECR1, AMMECR1 (S) were both localized in the nucleus (Figure 2).

\subsection{Phosphorylation of AMMECR1 at Serine 16}

The phosphorylation of protein is a ubiquitous and extensively studied post-translational modification, which can control its subcellular localization, its interaction with other molecules and thus its function [10]. We try to investigate the phosphorylation of AMMECR1. Bioinformatics information shows that there are six phosphorylation sites of Protein kinase C (PKC) in AMMECR1. With Phos-tag SDS PAGE analysis, the migration speed of phosphorylated proteins decreases and they can be separated from the non-phosphorylated forms. Using this technique, we showed that AMMECR1 was indeed phosphorylated,

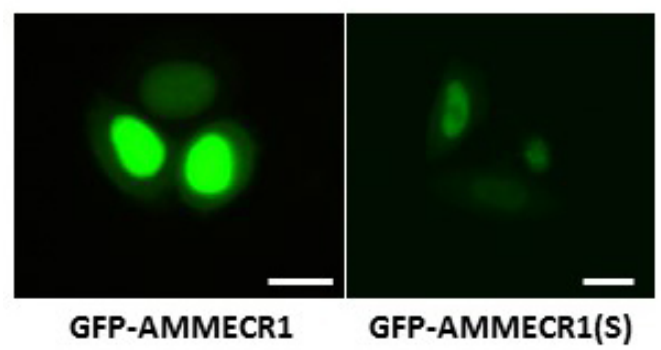

Figure 2. The nuclear location of AMMECR1 isoforms in HeLa cells. HeLa Cells were transiently transfected with $2 \mu \mathrm{g}$ of plasmid DNA pcDNA3-GFP-AMMECR1, AMMECR1 (S), respectively. After $24 \mathrm{~h}$, the cells were examined under fluorescent microscope. Each scale bar represents $10 \mu \mathrm{m}$. 
and it got dephosphorylated by $\lambda$ PPase. However, its phosphorylation did not require the stimulation of PKC activator, TPA (Figure 3(A)).

Compared with other AMMECR1 homologs, human AMMECR1 has a unique N-terminal amino acid sequence (1-123) [2]. As the putative phosphorylation sites (S121, T150, T163, S249, T291 and S299) by PKC are located at C-terminal AMMECR1, we then examined whether the $\mathrm{N}$ - or C-terminus of AMMECR1 was phosphorylated. We found that AMMECR1 (1-123), but not AMMECR1 (124-333) was phosphorylated (Figure 3(B)), which is consistent with why the PKC activator cannot stimulate the phosphorylation of AMMECR1. Phosphoproteomic screening showed that there are six putative phosphorylation sites in AMMECR1 (S15, S16, S19, S21, S29 and S34) [11] [12] [13] [14]. We then mutated each of them to alanine, and transfected the mutants into HEK293T cells, respectively. The results showed that only the S16A mutation eliminated the phosphorylation of AMMECR1 (Figure 3(C)).

A

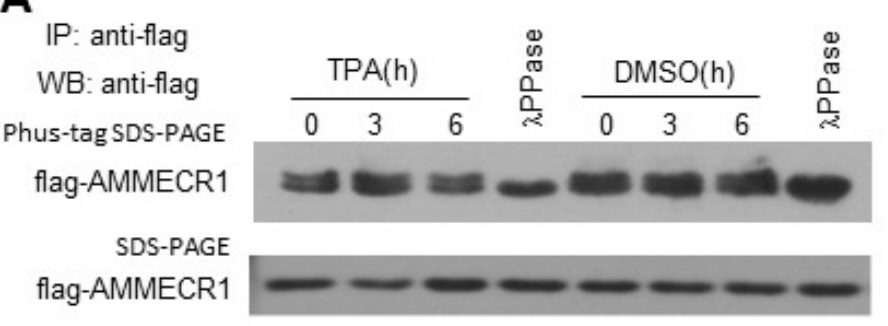

B

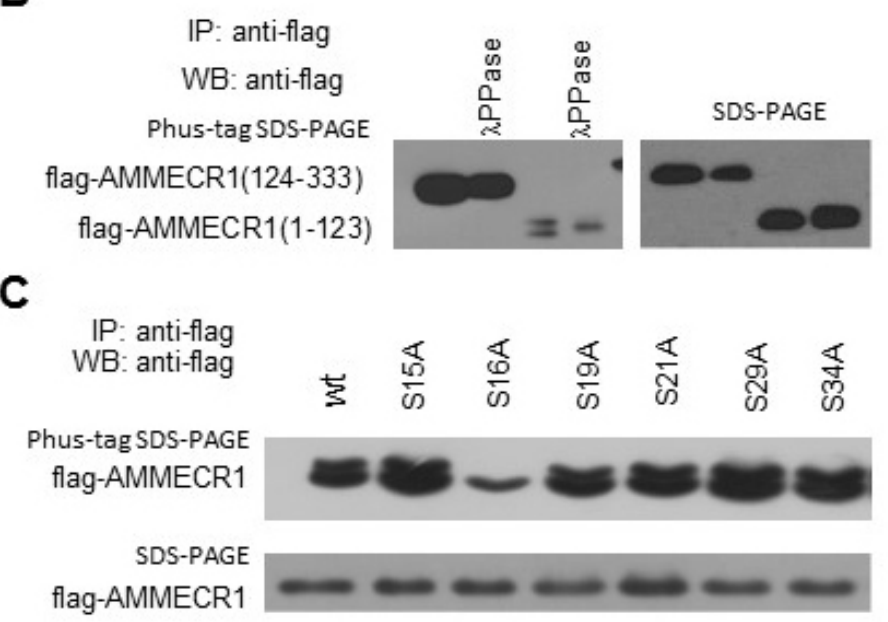

Figure 3. Phos-tag-based mobility shift detection of the phosphorylation of AMMECR1. HEK HEK293T cells were transfected with pcDNA3-flag: (A) AMMECR1 treated with or without TPA or DMSO; (B) AMMECR1 (1-123), AMMECR1 (124-333); (C) AMMECR1, AMMECR1 (S15A), AMMECR1 (S16A), AMMECR1 (S19A), AMMECR1 (S21A), AMMECR1 (S29A), AMMECR1 (S34A). The cells were harvested $24 \mathrm{~h}$ after transfection. Cell lysates were subjected to immunoprecipitation with anti-flag antibodies. The precipitates, treated with or without $\lambda$ PPase, were separated with acrylamide pendant Phos-tag SDS-PAGE or conventional SDS PAGE, and analyzed by Western blotting with anti-flag antibodies as indicated. 


\subsection{The Nuclear Localization of AMMECR1 Was Independent of Phosphorylation of AMMECR1}

Then, we want to know whether the nuclear localization of AMMECR1 was regulated by its phosphorylation. As shown in Figure 4(A), AMMECR1 (S16A) displayed nuclear localization, thus the phosphorylation of S16 was not required for AMMECR1 subcellular localization.

As mentioned before, AMMECR1 has a unique N-terminus, S16 lies in this region. AMMECR1 (124-333) is well conserved throughout the course of evolution from human and yeast to various microorganisms. We found that AMMECR1 (124-333) displayed nuclear localization (Figure 4(B)). AMMECR1 orthologue SPAC688.03c ( $S$. pombe) and MTH857 (Methanobacterium thermoautotrophicum) are highly homologous to AMMECR1 (124-333). Like AMMECR1 (124-333), both SPAC688.03c and MTH857 were also localized in the nucleus when expressed in HeLa cells (Figure 5(A)) and not phosphorylated (Figure 5(B)). Therefore, the phosphorylation was not essential to the nuclear localization of AMMECR1 homologues from different species. MTH857 is a prokaryotic archaeal protein but localized in the nucleus when expressed in

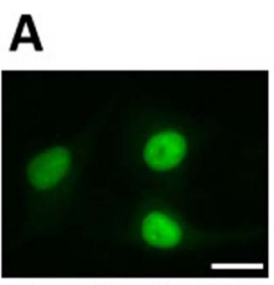

AMMECR1(S16A)
B

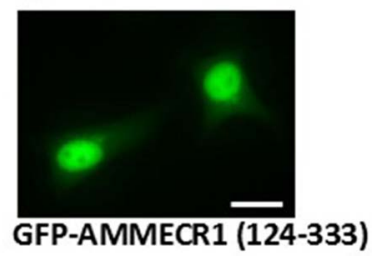

Figure 4. The nuclear location of AMMECR1 in HeLa cells. HeLa Cells were transiently transfected with $2 \mu \mathrm{g}$ of plasmid DNA pcDNA3-GFP: (A) AMMECR1 (S16A); (B) AMMECR1 (124-333), respectively. After $24 \mathrm{~h}$, the cells were examined under fluorescent microscope. Each scale bar represents $10 \mu \mathrm{m}$.

A

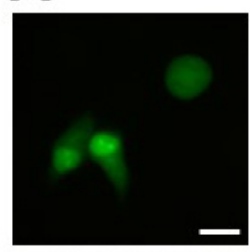

GFP-SPAC688

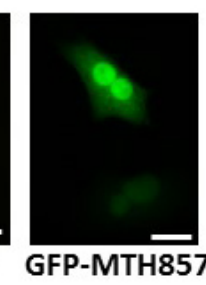

B

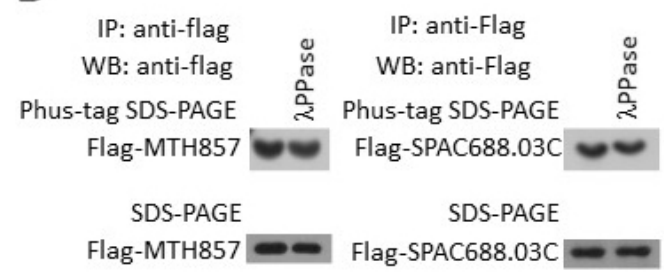

Figure 5. The nuclear location of MTH857 and SPAC688.03c in HeLa cells. (A) HeLa Cells were transiently transfected with $2 \mu \mathrm{g}$ of plasmid DNA pcDNA3-GFP-MTH857 or SPAC688.03c, respectively. After $24 \mathrm{~h}$, the cells were examined under fluorescent microscope. Each scale bar represents $10 \mu \mathrm{m}$; (B) HEK293T Cells in 6-cm plate were transiently transfected with $2 \mu \mathrm{g}$ of plasmid DNA pcDNA3-flag-MTH857, pcDNA3-flag-SPAC688.03c respectively. The cells were harvested $24 \mathrm{~h}$ after transfection. The cell lysates were subjected to immunoprecipitation with anti-flag antibodies. The precipitates, treated with or without $\lambda$ PPase, were separated with acrylamide pendant Phos-tag SDS-PAGE or conventional SDS PAGE, and analyzed by Western blotting with anti-flag antibodies as indicated. 
eukaryotic cells, so it is possible that AMMECR1 family proteins might have strong nuclear localization capability and can diffuse through the nuclear pore complexes due to its smaller molecular weight $(\sim 35.5 \mathrm{kDa})$ [13].

In summary, we found that many human cell lines expressed AMMECR1. AMMECR1 was phosphorylated only at S16 constitutively. AMMECR1 has no special nuclear localization signal but was a nuclear localization protein. S16A mutation did not affect its nuclear localization.

There is no doubt that the functional study of AMMECR1 requires a lot of hard and basic experimental work. Only in this way can the biological function of AMMECR1 be gradually revealed, which may be helpful for the detection of certain diseases in human.

\section{Acknowledgements}

This work was supported in part by Grants NSFC 31070688 from Chinese National Science Foundation and 2012AA02A201 from Ministry of Science and Technology of China; Grant from Open Research Fund of State Key Laboratory of Large Yellow Croaker Breeding, China (LYC2016RS01).

\section{Conflicts of Interest}

The authors declare no conflicts of interest regarding the publication of this paper.

\section{References}

[1] Jonsson, J.J., Renieri, A., Gallagher, P.G., Kashtan, C.E., Cherniske, E.M., Bruttini, M., et al. (1998) Alport Syndrome, Mental Retardation, Midface Hypoplasia, and Elliptocytosis: A New X Linked Contiguous Gene Deletion Syndrome? J Med Genet, 35, 273-278. https://doi.org/10.1136/jmg.35.4.273

[2] Vitelli, F., Piccini, M., Caroli, F., Franco, B., Malandrini, A., Pober, B., et al. (1999) Identification and Characterization of a Highly Conserved Protein Absent in the Alport Syndrome (A), Mental Retardation (M), Midface Hypoplasia (M), and Elliptocytosis (E) Contiguous Gene Deletion Syndrome (AMME). Genomics, 55, 335-340. https://doi.org/10.1006/geno.1998.5666

[3] Andreoletti, G., Seaby, E.G., Dewing, J.M., O’Kelly, I., Lachlan, K., Gilbert, R.D., et al. (2017) AMMECR1: A Single Point Mutation Causes Developmental Delay, Midface Hypoplasia and Elliptocytosis. JMed Genet, 54, 269-277.

https://doi.org/10.1136/jmedgenet-2016-104100

[4] Moysés-Oliveira, M., Giannuzzi, G., Fish, R.J., Rosenfeld, J.A., Petit, F., Soares, M.F., et al. (2018) Inactivation of AMMECR1 Is Associated with Growth, Bone, and Heart Alterations. Hum Mutat, 39, 281-291. https://doi.org/10.1002/humu.23373

[5] Basel-Vanagaite, L., Pillar, N., Isakov, O., Smirin-Yosef, P., Lagovsky, I., Orenstein, N., et al. (2017) X-Linked Elliptocytosis with Impaired Growth Is Related to $\mathrm{Mu}$ tated AMMECR1. Gene, 606, 47-52. https://doi.org/10.1016/j.gene.2017.01.001

[6] Jee, Y.H., Baron, J. and Nilsson, O. (2018) New Developments in the Genetic Diagnosis of Short Stature. Curr Opin Pediatr, 30, 541-547. https://doi.org/10.1097/MOP.0000000000000653 
[7] Dubois, A., Deuve, J.L., Navarro, P., Merzouk, S., Pichard, S., Commere, P.H., et al. (2014) Spontaneous Reactivation of Clusters of X-Linked Genes Is Associated with the Plasticity of X-Inactivation in Mouse Trophoblast Stem Cells. Stem Cells, 32, 377-390. https://doi.org/10.1002/stem.1557

[8] Nardozzi, J.D., Lott, K. and Cingolani, G. (2010) Phosphorylation Meets Nuclear Import: A Review. Cell Commun Signal, 8, 32. https://doi.org/10.1186/1478-811X-8-32

[9] Von Morgen, P., Lidak, T., Horejsi, Z. and Macurek, L. (2018) Nuclear Localisation of 53BP1 Is Regulated by Phosphorylation of the Nuclear Localisation Signal. Biol Cell, 110, 137-146. https://doi.org/10.1111/boc.201700067

[10] Grindheim, A.K., Saraste, J. and Vedeler, A. (2017) Protein Phosphorylation and Its Role in the Regulation of Annexin A2 Function. Biochim Biophys Acta Gen Subj, 1861, 2515-2529. https://doi.org/10.1016/j.bbagen.2017.08.024

[11] Huttlin, E.L., Jedrychowski, M.P., Elias, J.E., Goswami, T., Rad, R., Beausoleil, S.A., et al. (2010) A Tissue-Specific Atlas of Mouse Protein Phosphorylation and Expression. Cell, 143, 1174-1189. https://doi.org/10.1016/j.cell.2010.12.001

[12] Kettenbach, A.N., Schweppe, D.K., Faherty, B.K., Pechenick, D., Pletnev, A.A. and Gerber, S.A. (2011) Quantitative Phosphoproteomics Identifies Substrates and Functional Modules of Aurora and Polo-Like Kinase Activities in Mitotic Cells. Sci Signal, 4, rs5. https://doi.org/10.1126/scisignal.2001497

[13] Wu, X., Tian, L., Li, J., Zhang, Y., Han, V., Li, Y., et al. (2012) Investigation of Receptor Interacting protein (RIP3)-Dependent Protein Phosphorylation by Quantitative Phosphoproteomics. Mol Cell Proteomics, 11, 1640-1651. https://doi.org/10.1074/mcp.M112.019091

[14] Zhou, H., Di Palma, S., Preisinger, C., Peng, M., Polat, A.N., Heck, A.J., et al. (2013) Toward a Comprehensive Characterization of a Human Cancer Cell Phosphoproteome. J Proteome Res, 12, 260-271. https://doi.org/10.1021/pr300630k 